\title{
Moving Past the Routine Use of Macrolides-Reviewing the Role of Combination Therapy in Community-Acquired Pneumonia
}

\author{
Geoffrey Shumilak ${ }^{1} \cdot$ Wendy I. Sligl ${ }^{2}$ \\ Published online: 6 September 2018 \\ (C) Springer Science+Business Media, LLC, part of Springer Nature 2018
}

\begin{abstract}
Purpose of Review Despite advances in diagnostic microbiology and sepsis management, community-acquired pneumonia (CAP) remains a significant cause of morbidity and mortality. Current recommendations regarding the use of beta-lactams in combination with macrolides published in clinical practice guidelines are variable and based on low-quality evidence that is frequently retrospective, observational, and heterogeneous in nature. While population-based studies have historically suggested improved clinical outcomes with the routine use of macrolide combination therapy in hospitalized patients with CAP, emerging evidence from recent randomized controlled trials has challenged this practice. In this article, we discuss the historical rationale and current evidence for combination macrolide therapy in the management of CAP.

Recent Findings Recent randomized controlled trials have assessed the non-inferiority of beta-lactam monotherapy compared to beta-lactam/macrolide combination therapy in adult patients hospitalized with CAP. Beta-lactam monotherapy was associated with equivalent clinical outcomes in patients with mild to moderate CAP. Patients with severe CAP managed with beta-lactam monotherapy have demonstrated worse clinical outcomes when compared to patients treated with combination therapy. In addition, previous beta-lactam exposure prior to hospitalization has not been shown to negatively impact outcomes in patients managed with beta-lactam monotherapy in the hospital.

Summary Current evidence supports the use of beta-lactam monotherapy in adult patients hospitalized with mild to moderate CAP. While existing evidence supports the use of combination therapy in patients with severe pneumonia, further large-scale randomized controlled trials are urgently needed to clarify the role of combination therapy in this population.
\end{abstract}

Keywords Pneumonia $\cdot$ Community-acquired pneumonia $\cdot$ Antibiotic therapy $\cdot$ Macrolides $\cdot$ Combination therapy

\section{Introduction}

Community-acquired pneumonia (CAP) remains a significant cause of morbidity and mortality, resulting in an estimated 1.5 million adult hospitalizations annually in the United States $[1 \cdot 2]$. Despite advances in sepsis recognition and management and being a core measure by the Centers for Medicare \&

This article is part of the Topical Collection on Respiratory Infections

Geoffrey Shumilak

shumilak@ualberta.ca

1 Department of Critical Care Medicine, University of Alberta, 2-124 Clinical Sciences Building, 8440-112 Street, Edmonton, AB T6G 2B7, Canada

2 Department of Critical Care Medicine and Division of Infectious Diseases, University of Alberta, Edmonton, AB, Canada
Medicaid Services, the in-hospital mortality of patients admitted with CAP remains high at $6.5 \%$ with all-cause mortality at 30 days, 6 months, and 1 year estimated to be $13 \%, 23 \%$, and $31 \%$, respectively $[1 \cdot 3]$. The broad spectrum of pathogens causing CAP remains unchanged and includes typical bacteria (Streptococcus pneumoniae, Haemophilus influenzae, Moraxella catarrhalis), atypical bacteria (Mycoplasma pneumoniae, Chlamydia pneumoniae, Legionella spp.), viruses (such as rhinovirus, influenza, parainfluenza, respiratory syncytial virus, coronaviruses, human metapneumovirus) and less frequently Staphylococcus aureus, gram-negative bacilli, fungi, and mycobacteria [4]. While diagnostic techniques to facilitate the accurate identification of causative agents have improved, including the rapid evolution of commercial multiplex PCR assays, an etiologic agent is identified in fewer than half of CAP cases and frequently leaves clinicians to rely on empiric antimicrobials for management $[5,6 \bullet]$. 


\section{Mechanism of Action}

Macrolide antimicrobials are commonly prescribed agents for the management of respiratory tract infections [7]. As a class, macrolides are bacteriostatic and function by inhibiting bacterial protein synthesis through reversible binding to $23 \mathrm{~S}$ ribosomal RNA of the 50S ribosomal subunit, resulting in inhibition of RNA-dependent protein synthesis [8].

However, widespread resistance to macrolides due to point mutations in the $23 \mathrm{~S}$ rRNA binding site has significantly limited their utility $[9,10]$. S. pneumoniae resistance to macrolides has demonstrated regional variability with rates ranging from $30 \%$ in North America to over $90 \%$ in Vietnam while $M$. pneumoniae demonstrates similar resistance ranging from $10 \%$ in the United States to over $90 \%$ in regions of Japan [11-14].

Aside from antimicrobial properties, macrolides are also prescribed for their immune modulating effects, particularly in the setting of chronic inflammatory lung diseases. Macrolides have been shown to suppress proinflammatory cytokines [15], decrease polymorphonuclear cell recruitment [16], attenuate reactive oxygen species [17], and alter key transcription factors [18]. While several small studies have shown that immune modulation from macrolides may also be beneficial in acute CAP by reducing inflammatory mediators, supportive research linked to clinical outcomes remains limited and a clear benefit has not been established $[19,20]$.

\section{CAP Microbiology}

A recent effort to characterize causative CAP pathogens through the use of modern diagnostic tests has been published in the Center for Disease Control and Prevention (CDC) Etiology of Pneumonia in the Community (EPIC) study [6•]. The CDC EPIC study was a prospective, multicenter, population-based active surveillance study of 2320 patients with radiographically confirmed pneumonia at five US hospitals between 2010 and 2012. In addition to conventional diagnostic tests, extensive molecular and serologic testing was employed to characterize causative pathogens. In this cohort, human rhinovirus, influenza, and $S$. pneumoniae were the most common etiologic agents. Atypical pathogens were infrequently identifiedwith the incidence of $M$. pneumoniae, $C$. pneumoniae, and L. pneumophila each representing less than 0.5 cases per 10,000 adults in the community. This study provides contemporary, comprehensive microbiologic CAP data that is essential in informing guidelines and choosing empiric therapies wisely. The low incidence of atypical pathogens is of particular interest.
Clinical Practice Guideline Recommendations

Published recommendations regarding the use of beta-lactams in combination with macrolides in current clinical practice guidelines are variable and based on relatively low quality evidence that is frequently retrospective, observational, and heterogeneous in nature. Last updated in 2007, the joint Infectious Disease Society of America (IDSA)/American Thoracic Society (ATS) guidelines for CAP recommend empiric combination therapy with a beta-lactam plus macrolide or monotherapy with a respiratory fluoroquinolone (e.g., moxifloxacin) for adult patients hospitalized with CAP in a non-ICU setting [21]. Combination therapy is recommended for all patients with severe CAP (inpatient ICU). In contrast, guidelines published by the British Thoracic Society (BTS, 2009) and National Institute for Health and Care Excellence (NICE, 2014) recommend beta-lactam monotherapy for lowseverity CAP with consideration of combination therapy only in moderate to severe CAP $[22,23]$.

\section{Rationale for the Use of Macrolides}

The body of evidence used to support current IDSA/ATS guideline recommendations that advocate for combination therapy with a beta-lactam plus macrolide in the management of hospitalized adult patients with CAP originates from a series of large, retrospective cohort studies that showed improved clinical outcomes in patients treated with combination therapy.

Gleason et al. evaluated the relationship between initial antimicrobial therapy and clinical outcomes in a retrospective cohort of 12,945 Medicare patients hospitalized with pneumonia [24]. Thirty-day mortality was lower when a macrolide was combined with a second-generation cephalosporin (HR, $0.71 ; 95 \% \mathrm{CI}, 0.52-0.96)$ or third-generation cephalosporin (HR, 0.74; 95\% CI, 0.60-0.92) compared to conventional beta-lactam monotherapy. Houck et al. similarly evaluated a retrospective cohort of Medicare patients hospitalized with pneumonia in 10 western states during the years 1993, 1995, and 1997 [25]. Therapy with a macrolide in combination with a beta-lactam was associated with a significant reduction in mortality (aOR, 0.42 ; 95\% CI, 0.25-0.69) in 1993 but this effect was not observed during the other years of study. Dudas et al. subsequently performed a prospective, observational cohort study at 72 community hospitals to evaluate compliance with ATS guidelines in patients with non-severe community-acquired pneumonia and compared outcomes between patients administered varying combinations of antimicrobials [26]. Compliance with ATS guidelines was found to be $81 \%$ and the addition of a macrolide to a beta-lactam was associated with decreased mortality and reduced length of hospital stay. Brown et al. further reinforced these findings 
by evaluating a retrospective cohort of 44,814 patients in a hospital claims database [27]. In this cohort, macrolide combination therapy was associated with decreased mortality, length of hospital stay, and healthcare-associated costs overall.

Based on the findings of these large observational studies, many clinical practice guidelines recommend combination therapy with a beta-lactam plus macrolide or monotherapy with a respiratory fluoroquinolone as first-line therapy for hospitalized adult patients with CAP. However, this recommendation for routine combination therapy for the management of CAP has remained a contentious issue.

Despite the body of (predominantly retrospective, observational) evidence that demonstrates improved clinical outcomes, many clinicians regard this evidence (which grounds guideline recommendations) to be low quality and have called for further prospective, randomized-controlled trials to address this question. The routine use of macrolides in hospitalized adults to augment the spectrum of antimicrobial coverage in mild to moderate CAP patients to include atypical pathogens has been questioned owing to the infrequent identification of these pathogens. M. pneumoniae and C. pneumoniae are also specifically known for producing relatively mild illness with minimal existing literature that targeted antibiotic therapy impacts patient mortality [28]. Additionally, any adjunctive benefit from the postulated anti-inflammatory effect of macrolides in the treatment of CAP remains unproven. Given the increasing resistance of $S$. pneumoniae and $M$. pneumoniae isolates to macrolide antibiotics, unnecessary exposure to potential harms associated with macrolide use (potential cardiotoxicity, Clostridium difficile infection, etc.), and the economic costs of unnecessary combination therapy, many have called the routine use of combination beta-lactams plus macrolides into question [29-33].

Additional studies have attempted to bring clarity to this controversy. A systematic review and meta-analysis performed by the Cochrane Acute Respiratory Infections Group explored if the addition of atypical coverage to an antimicrobial regimen would improve patient outcomes [34]. In 28 trials, including 5939 patients hospitalized with CAP, there was no overall difference in mortality (RR 1.14, 95\% CI: $0.84-1.55)$ between the atypical and the typical coverage arms. A trend towards fewer clinical failures was observed in the atypical therapy arm, but this did not achieve statistical significance (RR 0.93 ; 95\% CI: 0.84-1.04). Of note, the Cochrane Review only included one study that specifically focused on the comparison of beta-lactam monotherapy to beta-lactam/macrolide combination therapy. Asadi et al. also compared macrolides to other treatment regimens in adults hospitalized with CAP in a systematic review and metaanalysis including 23 studies and 137,574 patients [35]. While overall macrolide use was associated with a statistically significant reduction in mortality when compared to nonmacrolide use, the survival advantage disappeared (4.6 vs
4.1\%; RR 1.13; 95\% CI: 0.65-1.98) when analyses were restricted to RCTs.

\section{Randomized Trials}

In recent years, researchers have attempted to address the paucity of high-quality evidence with several prospective, randomized-controlled trials aimed at evaluating the utility of combination antimicrobial therapy in hospitalized adults with CAP.

Garin et al. tested the non-inferiority of beta-lactam monotherapy compared with beta-lactam/macrolide combination therapy in moderately severe patients with CAP in an openlabel, multicenter, non-inferiority randomized control trial [36••]. The study was conducted in six acute care hospitals in Switzerland from 2009 to 2013. The primary endpoint in this trial was the proportion of patients not achieving clinical stability at day 7 . After 7 days of therapy, $41 \%$ in the monotherapy arm and $33 \%$ in the combination arm had not reached clinical stability (7\% difference, $p=0.07$ ), exceeding the predefined non-inferiority boundary. Patients identified to have atypical pathogens or severe pneumonia (PSI $\geq 4$ ) were less likely to reach clinical stability with monotherapy while patients infected with typical pathogens or mild to moderate CAP severity (PSI 1 to 3 ) had equivalent outcomes. Secondary endpoints that included overall mortality, need for ICU admission, complications, length of stay, and recurrence of pneumonia within 90 days did not differ significantly between the two groups.

Postma et al. designed the CAP-START Trial, a multicenter cluster-randomized, crossover, non-inferiority trial conducted at 7 hospitals in the Netherlands to determine if empiric therapy for CAP with beta-lactam monotherapy was noninferior to combination beta-lactam/macrolide therapy or a respiratory fluoroquinolone $[37 \cdot \bullet]$. Adult patients were included if they demonstrated radiographic evidence of pneumonia and compatible clinical findings. Patients were excluded if they had cystic fibrosis, positive Legionella antigen, recent hospitalization, or were from a long-term care facility. The unadjusted 90-day all-cause mortality was $9.0 \%$ (betalactam monotherapy), $8.8 \%$ (FQ monotherapy), and $11.1 \%$ (beta-lactam/macrolide combination). Adjusted comparisons for 90-day all-cause mortality favored beta-lactam monotherapy with a $1.9 \%$ lower risk of death when compared to macrolide combination therapy ( $90 \%$ CI: -0.6 to 4.4 ). Time to oral antibiotic de-escalation, length of hospital stay, and occurrence of complications during hospital stay were not significantly different between the groups. While criticisms of this study included a high rate of protocol deviation in the beta-lactam monotherapy group to include atypical coverage, subgroup analyses of strategy-adherent and antibiotic- 
Table 1 Clinical practice guideline recommendations for empiric management of CAP in hospitalized adult patients (Immunocompetent)

\begin{tabular}{|c|c|c|c|}
\hline & IDSA/ATS (2007) & BTS (2009) & NICE (2014) \\
\hline Mild CAP & $\begin{array}{l}\text { Respiratory fluoroquinolone } \\
\text { or } \beta \text {-lactam plus macrolide }\end{array}$ & $\begin{array}{l}\beta \text {-lactam monotherapy } \\
\quad(\text { amoxicillin preferred })\end{array}$ & $\begin{array}{l}\beta \text {-lactam monotherapy } \\
\quad(\text { amoxicillin preferred) }\end{array}$ \\
\hline $\begin{array}{l}\text { Moderate } \\
\text { CAP }\end{array}$ & $\begin{array}{l}\text { Respiratory fluoroquinolone } \\
\text { or } \beta \text {-lactam plus macrolide }\end{array}$ & $\begin{array}{l}\beta \text {-lactam plus macrolide or } \\
\text { doxycycline or respiratory fluoroquinolone }\end{array}$ & $\begin{array}{l}\beta \text {-lactam plus macrolide } \\
\quad \text { (amoxicillin preferred) }\end{array}$ \\
\hline $\begin{array}{l}\text { Severe } \\
\text { CAP }\end{array}$ & $\begin{array}{l}\beta \text {-lactam plus macrolide } \\
\text { or respiratory fluoroquinolone }\end{array}$ & $\beta$-lactam plus macrolide & $\begin{array}{l}\beta \text {-lactam plus macrolide } \\
\quad(\beta \text {-lactamase stable agent })\end{array}$ \\
\hline
\end{tabular}

adherent groups revealed no significant difference in outcomes.

Van Werkhoven et al. further evaluated outcomes in a subset of 179 patients from the CAP-START Trial that had previous beta-lactam exposure prior to hospitalization [38••]. Thirty-day mortality, hospital length of stay, and frequency of treatment escalations were compared between patients that were continued on beta-lactam monotherapy and patients that received beta-lactams and atypical coverage. Despite the betalactam monotherapy group being older, having more medical comorbidities, and longer duration of symptoms prior to admission, the authors found no significant differences between groups with respect to 30-day mortality and hospital length of stay.

While these recent trials performed by Garin, Postma, and van Werkhoven provide evidence to support a strategy of beta-lactam monotherapy for patients presenting with mild to moderate CAP, the role of combination beta-lactam/ macrolide therapy in critically ill patients with severe CAP remains unclear.

\section{Severe CAP}

The use of macrolide combination therapy in severe CAP is endorsed in various clinical guidelines (Table 1). Again, however, the data to support macrolide use is of relatively low quality and does not clearly explain whether improved outcomes in this population are derived from the antimicrobial or immune-modulatory properties of these agents.

Baddour et al. previously evaluated the impact of combination antibiotic therapy on 844 adult patients with severe bacteremic pneumococcal pneumonia in a prospective, observational multicenter study [39]. While overall 14-day mortality did not differ significantly in this cohort, combination therapy was associated with significantly reduced mortality among a subset of critically ill patients $(23.4$ vs $55.3 \%, p<$ 0.01 ) regardless of nationality, level of ICU support, class of antibiotics, and in vitro activity of the prescribed antibiotics. While this study was not limited to combination macrolide therapy, beta-lactam/macrolide therapy remained the most common regimen in the study population.
Multiple retrospective cohort studies have shown a similar effect in critically ill patients with severe pneumococcal pneumonia complicated by bacteremia [40]. In addition, Sligl et al. explored the impact of beta-lactam/macrolide combination therapy in critically ill patients in a systematic review and meta-analysis involving nearly 10,000 critically ill patients from 28 observational studies and found that combination therapy including macrolides was associated with a significant reduction in mortality [41]. A subgroup analysis of the cohort evaluated by Garin et al. also supports this literature - demonstrating that patients with severe pneumonia were less likely to achieve clinical stability with beta-lactam monotherapy.

Despite the lack of randomized trials in severe CAP, the available evidence is consistent with current recommendations published in the joint IDSA/ATS guidelines for management of CAP in patients requiring ICU admission. Further research to characterize the potential immune-modulatory effects of macrolides in patients with severe CAP is warranted, specifically because this population is more likely to have concomitant sepsis and deleterious systemic inflammation.

\section{Conclusions}

The utility of macrolide combination therapy in the management of CAP has been a source of significant controversy with a large body of conflicting evidence. Considering the increasing resistance of typical bacterial isolates (specifically $S$. pneumoniae) to macrolides and the infrequent identification of atypical pathogens as causative agents of pneumonia, clinicians need to carefully consider the utility of macrolides in the treatment of CAP. In addition, clinicians need to balance any potential incremental benefit from the anti-inflammatory effect that macrolides may possess against the urgent need for antimicrobial stewardship and responsible antibiotic use.

While population-based epidemiologic studies have historically suggested a benefit of adjunctive macrolides in the management of CAP, most of these studies are of relatively low quality. Emerging evidence from several prospective studies has challenged this practice by failing to show a significant improvement in relevant clinical outcomes in patients hospitalized with mild to moderate CAP. While existing evidence 
still supports the use of combination therapy in critically ill patients with severe CAP, further randomized-controlled trials are urgently needed to clarify the role of combination therapy in this population. In particular, the potential immune modulatory effect of macrolides in sepsis remains an area of uncertainty.

Based on the current literature, we suggest a pragmatic approach pending further high quality evidence to guide therapy. For patients with mild-moderate CAP requiring hospitalization, we suggest treatment with beta-lactam monotherapy (e.g., ceftriaxone) or a respiratory fluoroquinolone (e.g., moxifloxacin). For patients with severe pneumonia or those requiring ICU-level care, we suggest beta-lactam/macrolide (e.g., ceftriaxone/azithromycin) combination therapy. Empiric therapies, however, may differ based on local epidemiology and antimicrobial susceptibility patterns in each individual health region or institution.

\section{Compliance with Ethical Standards}

Conflict of Interest Geoffrey Shumilak and Wendy Sligl declare that they have no conflicts of interest.

Human and Animal Rights and Informed Consent This article does not contain any studies with human or animal subjects performed by any of the authors.

\section{References}

Papers of particular interest, published recently, have been highlighted as:

- Of importance

•- Of major importance

1. Ramirez JA, Wiemken TL, Peyrani P, Arnold FW, Kelley R, Mattingly WA, et al. Adults hospitalized with pneumonia in the United States: incidence, epidemiology, and mortality. Clin Infect Dis. 2017;65(11):1806-12. https://doi.org/10.1093/cid/cix647. Prospective population-based cohort study evaluating incidence of CAP in consecutive hospitalized patients at all adult hospitals in Louisville, Kentucky. Calculation of annual population-based incidence of CAP, mortality at 1, 6, and 12 months following hospitalization, and ecological associations of CAP.

2. Rui P, Kang K. National Hospital Ambulatory Medical Care Survey: 2014 Emergency department summary tables. Available from: http://www.cdc.gov/nchs/data/ahcd/nhamcs_emergency/ 2014 ed web tables.pdf.

3. Kochanek KD, Murphy SL, Xu JQ, Tejada-Vera B. Deaths: final for 2014. National vital statistics reports; vol 65 no4. National Center for Health Statistics: Hyattsville; 2016.

4. Bennett J, Dolin R, Mandell BM. Douglas and Bennett's Principles and Practice of Infectious Diseases. Chapter 68: Acute Pneumonia. Philadelphia: Elsevier; 2015. p. 823-828.

5. Mandell LA, Marrie TJ, Grossman RF, Chow AW, Hyland RH, The Canadian CAP Working Group. Summary of Canadian Guidelines for the Initial Management of Community-acquired Pneumonia: an evidence-based update by the Canadian Infectious Disease Society and the Canadian Thoracic Society. Can J Infect Dis. 2000;11(5): 237-48.

6. Jain S, Self WH, Wunderink RG, Fakhran S, Balk R, Bramley AM, et al. Community-acquired pneumonia requiring hospitalization among U. S. adults. N Engl J Med. 2015;373:415-27. https://doi. org/10.1056/NEJMoa.1500245. Active population-based surveillance study of immunocompetent adults hospitalized with community-acquired pneumonia. In addition to conventional diagnostic testing, extensive molecular and serologic testing was employed to characterize causative pathogens and provide contemporary data on the causative agents of CAP. Despite extensive testing, found that no pathogen was detected in the majority of patients. Rhinovirus, influenza, and Streptococcus pneumoniae remained the most common identifiable causes of CAP.

7. McFarlane A, Sligl W. The value of macrolide-based regimens for community-acquired pneumonia. Curr Infect Dis Rep. 2015;17:50. https://doi.org/10.1007/s11908-015-0507-4.

8. Schlunzen F, Zarivach R, Harms J, et al. Structural basis for the interaction of antibiotics with the peptidyl-transferase centre in eubacteria. Nature. 2001;413(6858):814-21. https://doi.org/10. $1038 / 35101544$.

9. Tenson T, Lovmar M, Ehrenberg M. The mechanism of action of macrolides, lincosamides and streptogramin B reveals the nascent peptide exit path in the ribosome. J Mol Biol. 2003;330(5):100514.

10. Doern GV, Richter SS, Miller A, Miller N, Rice C, Heilmann K, et al. Antimicrobial resistance among Streptococcus pneumoniae in the United States: have we begun to turn the corner on resistance to certain antimicrobial classes? Clin Infect Dis. 2005;41(2):139 48.

11. Centers for Disease Control and Prevention. Antibiotic Resistance Threats in the United States, 2013. https://www.cdc.gov/ drugresistance/threat-report-2013/ (Accessed on April 28, 2018).

12. Song JH, Jung SI, Ko KS, Kim NY, Son JS, Chang HH, et al. High prevalence of antimicrobial resistance among clinical Streptococcus pneumoniae isolates in Asia (an ANSORP study). Antimicrob Agents Chemother. 2004;48(6):2101-7.

13. Zheng X, Lee S, Selvarangan R, Qin X, Tang YW, Stiles J, et al. Macrolide-resistant mycoplasma pneumoniae, United States. Emerg Infect Dis. 2015;21(8):1470-2.

14. Kawai Y, Miyashita N, Kubo M, Akaike H, Kato A, Nishizawa Y, et al. Nationwide surveillance of macrolide-resistant mycoplasma pneumoniae infection in pediatric patients. Antimicrob Agents Chemother. 2013;57(8):4046-9.

15. Rubin BK, Tamaoki J. Macrolide antibiotics as biological response modifiers. Curr Opin Investig Drugs. 2000;1(2):169-72.

16. Khair OA, Devalia JL, Abdelaziz MM, Sapsford RJ, Davies RJ. Effect of erythromycin on Haemophilus influenzae endotoxininduced release of IL-6, IL- 8 and sICAM-1 by cultured human bronchial epithelial cells. Eur Respir J. 1995;8(9):1451-7.

17. Anderson R, Theron AJ, Feldman C. Membrane-stabilizing, antiinflammatory interactions of macrolides with human neutrophils. Inflammation. 1996;20(6):693-705.

18. Desaki M, Okazaki H, Sunazuka T, Omura S, Yamamoto K, Takizawa H. Molecular mechanisms of anti-inflammatory action of erythromycin in human bronchial epithelial cells: possible role in the signaling pathway that regulates nuclear factor-kappa B activation. Antimicrob Agents Chemother. 2004;48(5):1581-5.

19. Lee N, Wong C, Chan M, Yeung E, Tam W, Tsang O, et al. Antiinflammatory effects of adjunctive macrolide treatment in adults hospitalized with influenza: a randomized controlled trial. Antivir Res. 2017;144:48-56.

20. Kovaleva A, Remmelts HHF, Rijkers GT, Hoepelman AIM, Biesma DH, Oosterheert JJ. Immunomodulatory effects of 
macrolides during community-acquired pneumonia: a literature review. J Antimicrob Chemother. 67(3):530-40. https://doi.org/10. 1093/jac/dkr520.

21. Mandell LA, Wunderink RG, Anzueto A, Bartlett JG, Campbell GD, Dean NC, et al. Infectious Diseases Society of America/ American Thoracic Society consensus guidelines on the management of community-acquired pneumonia in adults. Clin Infect Dis. 2007;44 Suppl 2:S27-72.

22. Lim WS, Baudouin SV, George RC, Hill AT, Jamieson C, Le Jeune I, et al. BTS guidelines for the management of community acquired pneumonia in adults: update 2009. Thorax. 2009;64 Suppl 3:iii155. https://doi.org/10.1136/thx.2009.121434.

23. Sinan E, Celia P, Bernard H, Mark W. Diagnosis and management of community and hospital acquired pneumonia in adults: summary of NICE guidance. BMJ. 2014;349:g6722. https://doi.org/10.1136/ bmj.g6722.

24. Gleason PP, Meehan TP, Fine JM, Galusha DH, Fine MJ. Associations between initial antimicrobial therapy and medical outcomes for hospitalized elderly patients with pneumonia. Arch Intern Med. 159:1999, 2562-72.

25. Houck PM, MacLehose RF, Niederman MS, Lowery JK. Empiric antibiotic therapy and mortality among Medicare pneumonia inpatients in 10 western states: 1993, 1995, and 1997. Chest. 2001;119: 1420-6.

26. Dudas V, Hopefl A, Jacobs R, Guglielmo BJ. Antimicrobial selection for hospitalized patients with presumed community-acquired pneumonia: a survey of nonteaching US community hospitals. Ann Pharmacother. 2000;34:446-52

27. Brown RB, Iannini P, Gross $P$, Kunkel M. Impact of initial antibiotic choice on clinical outcomes in community-acquired pneumonia: analysis of a hospital claims-made database. Chest. 2003;123: $1503-11$.

28. Gardiner SJ, Gavranich JB, Chang AB. Antibiotics for communityacquired lower respiratory tract infections secondary to in children. Cochrane Database Syst Rev 2015, (1):CD004875. https://doi.org/ 10.1002/14651858.CD004875.pub5.

29. van Werkhoven CH, Postma DF, Oosterheert DF, Bonten MJM. Antibiotic treatment of moderate-severe community-acquired pneumonia: design and rationale of a multicenter cluster-randomized cross-over trial. Neth J Med. 2014;72(3)

30. Weiss K, Tillotson GS. The controversy of combination vs monotherapy in the treatment of hospitalized community-acquired pneumonia. Chest. 2005;128:940-6.

31. File TM Jr, Marrie TJ. Does empiric therapy for atypical pathogens improve outcomes for patients with CAP? Infect Dis Clin N Am. 2013;27:99-114.

32. Ray W, Murray K, Hall K, Arbogast P, et al. Azithromycin and the risk of cardiovascular death. N Engl J Med. 2012;366:1881-90. https://doi.org/10.1056/NEJMoa1003833.

33. Wieczorkiewicz J, Lopansri B, Cheknis A, Osmolski J, Hect D, Gerdin D, et al. Fluoroquinolone and macrolide exposure predict Clostridium difficile infection with the highly fluorquinolone and macrolide-resistant epidemic $C$. difficile strain BI/NAP1/027. Antimicrob Agents Chemother. 2016;60(1):418-23.

34. Eliakim-Raz N, Robenshtok E, Shefet D, et al. Empiric antibiotic coverage of atypical pathogens for community-acquired pneumonia in hospitalized adults. Cochrane Database Syst Rev. 2012;9: CD004418.

35. Asadi L, Sligl WI, Eurich DT, Colmers IN, Tjosvold L, Marrie TJ, et al. Macrolide-based regimens and mortality in hospitalized patients with community-acquired pneumonia: a systematic review and meta-analysis. Clin Infect Dis. 2012 Aug;55(3):371-80. https:// doi.org/10.1093/cid/cis414.

36.• Garin N, Genné D, Carballo S, Chuard C, Eich G, Hugli O, et al. $\beta$ Lactam monotherapy vs $\beta$-lactam-macrolide combination treatment in moderately severe community-acquired pneumonia: a randomized noninferiority trial. JAMA Intern Med. 2014;174(12): 1894-901. https://doi.org/10.1001/jamainternmed.2014.4887. Evaluated the non-inferiority of beta-lactam monotherapy compared with beta-lactam/macrolide combination therapy in moderately severe CAP in an open-label, multicenter, noninferiority randomized control trial. Failed to meet primary outcome and demonstrate non-inferiority of beta-lactam monotherapy in moderately severe community-acquired pneumonia. Subgroup analyses revealed equivalent outcomes in patients with mild to moderate CAP (PSI 1-3) but worse outcomes in patients managed with beta-lactam monotherapy and severe CAP. Secondary outcomes were equivalent between the two groups.

37.• Postma DF, van Werkhoven CH, van Elden LJR, et al. Antibiotic treatment strategies for community-acquired pneumonia in adults. (CAP-START). N Engl J Med. 2015;372:1312. Multi-center cluster-randomized, crossover, non-inferiority trial conducted at 7 hospitals in the Netherlands to determine if empiric therapy for CAP with beta-lactam monotherapy was non-inferior to combination beta-lactam/macrolide therapy or a respiratory fluoroquinolone. 90-day all-cause mortality was lowest in the betalactam monotherapy arm. Adjusted comparisons for 90-day all-cause mortality favored beta-lactam monotherapy with a $1.9 \%$ lower risk of death when compared to macrolide combination therapy. Time to oral antibiotic de-escalation, length of hospital stay, and occurrence of complications during hospital stay were not significantly different between the groups.

38.•v van Werkhoven CH, van de Garde EMW, Oosterheert JJ, Postma DF, Bonten MJM. Atypical coverage in community-acquired pneumonia after outpatient beta-lactam monotherapy. Respir Med. 2017;129:145-51. https://doi.org/10.1016/j.rmed.2017.06.012. Evaluation of outcomes in a subset of $\mathbf{1 7 9}$ patients from the CAP-START Trial that had previous beta-lactam exposure prior to hospitalization. Compared thirty-day mortality, hospital length of stay, and frequency of treatment escalations between patients that were continued on beta-lactam monotherapy and patients that received beta-lactams and atypical coverage. No significant differences between groups with respect to 30-day mortality and hospital length of stay were identified.

39. Baddour LM, Yu VL, Klugman KP, et al. Combination antibiotic therapy lowers mortality among severely ill patients with pneumococcal bacteremia. Am J Respir Crit Care Med. 2004;170:440-4.

40. Arnold FW, Lopardo G, Wiemken TL, Kelley R, Peyrani P, Mattingly WA, et al. Macrolide therapy is associated with lower mortality in community-acquired bacteremic pneumonia. Respir Med. 2018;140:115-21. https://doi.org/10.1016/j.rmed.2018.05. 020 .

41. Sligl WI, Asadi L, Eurich DT, Tjosvold L, Marrie TJ, Majumdar SR. Macrolides and mortality in critically ill patients with community-acquired pneumonia: a systematic review and metaanalysis. Crit Care Med. 2014;42(2):420-32. https://doi.org/10. 1097/CCM.0b013e3182a66b9b. 